ARTICLE

Received 8 Feb 2016 | Accepted 3 Oct 2016 | Published 10 Nov $2016 \quad$ DOl: 10.1038/ncomms13420 OPEN

\title{
Coupled binding mechanism of three sodium ions and aspartate in the glutamate transporter homologue Glt $\mathrm{T}_{\mathrm{Tk}}$
}

Albert Guskov $^{1, \star}$, Sonja Jensen ${ }^{1, \star}$, Ignacio Faustino ${ }^{1}$, Siewert J. Marrink ${ }^{1,2}$ \& Dirk Jan Slotboom,2

Glutamate transporters catalyse the thermodynamically unfavourable transport of anionic amino acids across the cell membrane by coupling it to the downhill transport of cations. This coupling mechanism is still poorly understood, in part because the available crystal structures of these transporters are of relatively low resolution. Here we solve crystal structures of the archaeal transporter $\mathrm{Glt}_{\mathrm{TK}}$ in the presence and absence of aspartate and use molecular dynamics simulations and binding assays to show how strict coupling between the binding of three sodium ions and aspartate takes place.

\footnotetext{
${ }^{1}$ University of Groningen, Groningen Biomolecular Sciences and Biotechnology Institute, Nijenborgh 4, 9747 AG Groningen, The Netherlands. ${ }^{2}$ University of Groningen, Zernike Institute for Advanced Materials, Nijenborgh 4, 9747 AG Groningen, The Netherlands. * These authors contributed equally to this work. Correspondence and requests for materials should be addressed to D.J.S. (email: d.j.slotboom@rug.nl).
} 
$\mathrm{G}$ lutamate transporters are found in Bacteria, Archaea and Eukarya. In vertebrates they guard the levels of the neurotransmitter glutamate in the synaptic cleft by pumping it (back) into neuronal and glial cells (reviewed in refs 1,2 ). The free energy stored in cation gradients across the membrane is utilized to accumulate the amino acid inside the cells. The eukaryotic proteins couple glutamate uptake to co-transport of three sodium ions and a proton, and countertransport of a potassium ion ${ }^{3-5}$.

Crystal structures are available for two similar archaeal homologues of the mammalian transporters $\left(\mathrm{Glt}_{\mathrm{Ph}}\right.$ and $\mathrm{Glt}_{\mathrm{Tk}}$, which share $77 \%$ sequence identity, Fig. 1a $)^{6-10}$. The archaeal transporters also couple the cellular uptake of aspartate to the co-transport of sodium ions, but they do not use protons or potassium ions for co- or counter-transport ${ }^{11}$. A stoichiometry of three sodium ions per aspartate has been determined for $\mathrm{Glt}_{\mathrm{Ph}}$ (ref. 12). The crystal structures have provided a structural framework to explore the mechanism of transport, which has sparked a large amount of biochemical, electrophysiological, mutagenesis and simulation studies on various members of the family. However, the resolution of the structural data has been too low to reveal the binding sites of the sodium ions, and therefore the coupling mechanism has remained poorly understood. Replacement of sodium by thallium ions allowed the use of the anomalous signal from the heavy atom to identify the potential locations of two of the three sodium binding sites in the low resolution structures ${ }^{9}$. Simulation studies indicated possible locations of the third sodium binding site ${ }^{13-15}$, but no unequivocal solution was found, leaving the coupling mechanism unexplained.

Here, we present crystal structures, molecular dynamics simulations and substrate binding assays of $\mathrm{Glt}_{\mathrm{Tk}}$ to localize the sodium binding sites, and provide insight in the mechanism of coupling between sodium and aspartate binding.

\section{Results}

Overall structural characteristics of $\mathbf{G l t}_{\mathbf{T k}}$. We crystallized the archaeal glutamate transporter homologue from Thermococcus kodakarensis $\left(\mathrm{Glt}_{\mathrm{Tk}}\right)$ in the presence of aspartate and sodium ions, solved its structure at $2.8 \AA$ resolution $\left(\mathrm{Gl}_{\mathrm{Tk}}^{\mathrm{sub}}\right)$, and located three sodium sites. Isothermal titration calorimetry (ITC) experiments on aspartate binding in the presence of eight different sodium concentrations showed that $\mathrm{Glt}_{\mathrm{Tk}}$ indeed binds three sodium ions per aspartate molecule, the same number as Glt $_{\mathrm{Ph}}$ (Fig. 2). In parallel we obtained improved crystals of the substrate-free form of $\mathrm{Glt}_{\mathrm{Tk}}$ (ref. 8) that yielded a $2.7 \AA$ resolution structure $\left(\mathrm{Gl}_{\mathrm{Tk}}^{\mathrm{apo}}\right)$ (see Table 1 for data and refinement statistics). The two structures, each with better resolution and data quality than any previously determined one, provide a structural model for coupling between substrate and cation transport.

Both structures have a very similar trimeric organization with the transport domains in an outward-occluded position (Supplementary Fig. 1). There was well-defined electron density for all loops, as well as for the N-termini, which were not ordered in the Glt $t_{\mathrm{Ph}}$ crystals. Notably, we could model the entire loop between transmembrane helices 3 and 4, which is important for transport $^{16,17}$ (Fig. 1b). This loop intimately wraps around the outer face of the transport domain, and appears to prevent excessive movements of helical hairpin 2 (HP2) away from the binding pocket, while allowing small-scale movements of HP2, which are required to provide access to the substrate and sodium binding sites (see below).

Sodium and aspartate binding sites. For all three sodium ions, as well as the aspartate molecule we observed well defined electron density in the Gltsub structure (Fig. $3 \mathrm{a}-\mathrm{c}$ ). The positions of sodium ions in the two sites $\mathrm{Na}^{+} 1$ and $\mathrm{Na}^{+} 2$ (numbering of the sodium ion sites as in ref. 9) are in good agreement with the previously reported crystal structures ${ }^{9}$, showing that thallium was indeed a good mimic for sodium in these sites (Supplementary

a

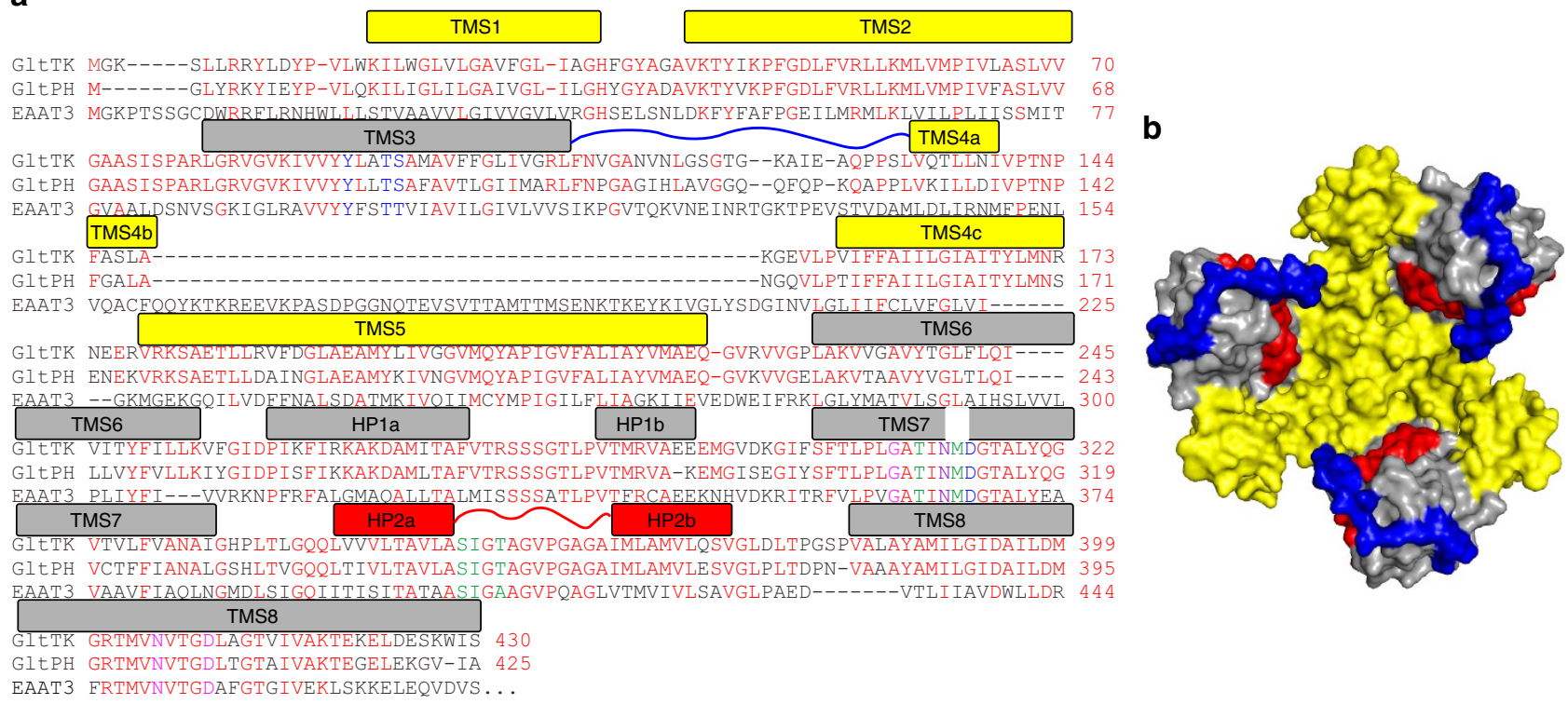

Figure 1 | Overview of the structure of $\mathbf{G l t}_{\mathbf{T k}}$. (a) Sequence alignment of the aspartate transporters Glt $\mathrm{T}_{\mathrm{Tk}}$ form Thermococcus kodakarensis and Glt $\mathrm{t}_{\mathrm{Ph}}$ from Pyrochoccocus horikoschii and the glutamate transporter EAAT3 from Rattus norvegicus. Identical residues between the archaeal proteins colored red, those involved in $\mathrm{Na}^{+} 1$ binding in magenta, $\mathrm{Na}^{+} 2$ in green and $\mathrm{Na}^{+} 3$ in blue respectively. $\mathrm{N} 313$ involved in coordination of both $\mathrm{Na}^{+} 1$ and $\mathrm{Na}+3$ is in purple. Bars above the sequences indicate helical segments and are colored in yellow and grey for the trimerization and transport domain, respectively. Loop 3-4 is indicated by the blue line, HP2 in red. (b) Crystal structure of the substrate-loaded aspartate transporter Glt $\mathrm{T}_{\mathrm{Tk}}^{\text {sub }}$ viewed from the extracellular side of the membrane, shown as surface. The trimerization domains in yellow, transport domains in gray, HP2 in red and long loop 3-4 in blue. 


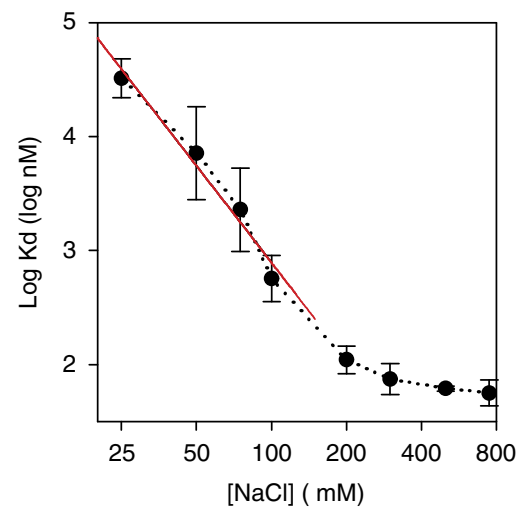

Figure 2 | Glt $_{\mathbf{T k}}$ binds three sodium ions per aspartate molecule.

Apparent affinity of $\mathrm{Glt}_{\mathrm{Tk}}$ for aspartate as a function of $\mathrm{Na}^{+}$concentration, as determined by isothermal titration calorimetry. In the limit of low sodium concentrations the negative slope of the plot approaches the $\mathrm{Na}^{+}$:aspartate binding stoichiometry $9,10,26$. The slope of the red line is -2.8 . Error bars indicate standard deviations from at least 3 independent experiments.

Fig. 2). $\mathrm{Na}^{+} 1$ is coordinated by the $\beta$-carboxylate of the conserved D409 residue (D405 in Glt $\mathrm{Ph}$ D454 in rat EAAT3, see Fig. 1a for numbering) of Trans Membrane Segment (TMS) 8, and mainchain carbonyls of G309, N313 from the unwound central region of TMS7 and N405 from TMS8 (Fig. 3a and Supplementary Fig. 3). $\mathrm{Na}^{+} 2$ is coordinated by main-chain carbonyls (S352, I353 and T355) of HP2 connecting TMS7 and 8, and main-chain carbonyl of T311 (TMS7) (Fig. 3a and Supplementary Fig. 3). The sulfur atom of M314 is within interacting distance $(<3 \AA)$ to $\mathrm{Na}^{+} 2$, though the nature of this interaction is unclear. The proposal made by Boudker et al. ${ }^{9}$ that the $\mathrm{Tl}^{+}$ion (used as a substitute of $\mathrm{Na}^{+}$) forms favorable interactions with sulfur is unlikely to be the full explanation, because we also see the interaction in the presence of sodium instead of thallium. The unusual coordination of $\mathrm{Na}^{+} 2$ by the methionine residue might be necessary for dynamic rearrangements of the binding site during the transport cycle. It was shown previously that the side chain of this methionine points away from the binding site in the substrate-free transporter, indicating that it must be highly mobile ${ }^{8}$. Furthermore, simulations have shown that $\mathrm{Na}^{+} 2$ is the last ion to bind during the substrate loading ${ }^{13,15}$ and the first one to leave for substrate release, suggesting that the $\mathrm{Na}^{+} 2$ binding site might be of low affinity, which is supported by simulations presented below.

The third sodium binding site. The third binding site is located between the unwound central region of helix 7 containing the essentially conserved NMDGT motif and TMS3, at a distance of $\sim 8 \AA$ from $\mathrm{Na}^{+} 1$, from which it is shielded by the side chain of $\mathrm{N} 313$ (Fig. 3c). $\mathrm{Na}^{+} 3$ is coordinated by hydroxyl groups of T94 (T92 in Glt ${ }_{\mathrm{Ph}}$, T101 in EAAT3 ${ }_{\text {rat }}$ ) and S95 (S93 in Glt $\mathrm{Ph}_{\mathrm{Ph}}$, T102 in EAAT3 $3_{\text {rat }}$ ) from TMS3; the carboxamide group of conserved

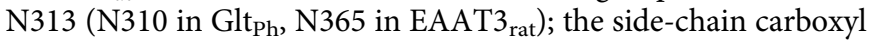
of D315 (D312 in Glt ${ }_{\mathrm{Ph}}, \mathrm{D} 367$ in EAAT3 ${ }_{\text {rat }}$ ); and the main-chain carbonyl of Y91 (Fig. 3 and Supplementary Fig. 3). The location of the third sodium binding site matches well with the site predicted in one of the published molecular dynamics simulations on $\mathrm{Glt}_{\mathrm{Ph}}$ (ref. 15), and with mutagenesis studies on mammalian transporters ${ }^{15,18-21}$.

Of the three sodium ions, the interaction distances are the shortest for the third ion $(\sim 2.2-2.4 \AA$ compared with $\sim 2.5-2.7 \AA$ for $\mathrm{Na}^{+} 1$ and $\mathrm{Na}^{+} 2$ ), which might indicate tighter binding of $\mathrm{Na}^{+} 3$ (Supplementary Table 1). Free energy calculations indeed show that sodium binds more tightly to the

\begin{tabular}{|c|c|c|}
\hline & $\mathbf{G I t}_{\mathbf{T K}}^{\text {apo }}$ & Glts sub \\
\hline \multicolumn{3}{|l|}{ Data collection } \\
\hline Space group & P3221 & \\
\hline \multicolumn{3}{|l|}{ Cell dimensions } \\
\hline$a, b, c(\AA)$ & 116.01116 .01308 .5 & \\
\hline$\alpha, \beta, \gamma\left(^{\circ}\right)$ & $90.00,90.00,120.00$ & \\
\hline Resolution $(\AA)$ & $47.7-2.7(2.78-2.7)^{\star}$ & $48.4-2.8(2.87-2.8)^{\star}$ \\
\hline$R_{\text {meas }}$ & $0.06(0.74)$ & $0.07(0.98)$ \\
\hline $\mathrm{CC}_{1 / 2}$ & $99.6(36.0)$ & $99.8(22.1)$ \\
\hline $\mid / \sigma l$ & $11(2.49)$ & $9.3(1.3)$ \\
\hline Completeness (\%) & $79(17.9)$ & $97.5(96.0)$ \\
\hline Redundancy & 3.4 & 3 \\
\hline \multicolumn{3}{|l|}{ Refinement } \\
\hline Resolution $(\AA)$ & 2.7 & 2.8 \\
\hline No. reflections & 52,820 & 60,568 \\
\hline$R_{\text {work }} / R_{\text {free }}(\%)$ & $19.8 / 23.7$ & $21.3 / 24.3$ \\
\hline \multicolumn{3}{|l|}{ No. atoms } \\
\hline Protein & 9,638 & 9,570 \\
\hline PEG/detergent & $278 / 40$ & $508 / 99$ \\
\hline Ligand/ion & - & $27 / 9$ \\
\hline Water & 60 & - \\
\hline \multicolumn{3}{|l|}{$B$-factors } \\
\hline Protein & 77.6 & 96.3 \\
\hline PEG/detergent & $114 / 132$ & $118.4 / 137.7$ \\
\hline Ligand/ion & - & $77.6 / 76.1$ \\
\hline Water & 69.9 & - \\
\hline \multicolumn{3}{|l|}{ r.m.s. deviations } \\
\hline Bond lengths $(\AA)$ & 0.008 & 0.008 \\
\hline Bond angles $\left(^{\circ}\right)$ & 1.123 & 1.131 \\
\hline
\end{tabular}

$\mathrm{Na}^{+} 3$ than to the two other sites (Supplementary Table 2 and ref. 22). The higher binding affinity may explain why thallium could not replace sodium in the heavy-atom soaking experiment in $\mathrm{Glt}_{\mathrm{Ph}}$ (ref. 9). Moreover, the observed interaction distances for $\mathrm{Na}^{+} 1$ and $\mathrm{Na}^{+} 2$ are more similar to those typical for potassium ions, and $\mathrm{Tl}^{+}$is a very good mimic for $\mathrm{K}^{+}$. It is also possible that steric hindrance prevented the larger $\mathrm{Tl}^{+}$ion (ionic radius of 1.64 versus $1.16 \AA$ for $\mathrm{Na}^{+}$) to enter the binding area for $\mathrm{Na}^{+} 3$.

Cooperativity between binding of sodium ions and aspartate. The affinity of $\mathrm{Glt}_{\mathrm{Ph}}$ and $\mathrm{Glt}_{\mathrm{Tk}}$ for aspartate is strongly dependent on the sodium concentration $8,9,23$. In the mammalian transporters similar cooperativity between sodium and glutamate has been observed. In addition, sodium binding to Glt $_{\mathrm{Ph}}$ in the absence of aspartate is also highly cooperative ${ }^{23,24}$. The structures of liganded $\mathrm{Glt}_{\mathrm{Tk}}^{\mathrm{sub}}$ and a new structure at $2.7 \AA$ resolution of $\mathrm{Gll}_{\mathrm{Tk}}^{\mathrm{apo}}$ provide a structural explanation for these observations. The most prominent difference between the two structures is the conformation of the conserved NMDGT motif in the central unwound region of TMS 7 (Fig. 4a), which is exemplified by the striking relocation of M314 from a position exposed to the lipid bilayer in the apo-state to the position as a ligand of $\mathrm{Na}^{+} 2$ in the substrate-bound state (Fig. 4a,c). Sodium binding to N313 plays a crucial role in stabilizing the repositioned NMDGT region. The side-chain of $\mathrm{N} 313$ coordinates $\mathrm{Na}^{+} 3$, while its main chain carbonyl binds $\mathrm{Na}^{+} 1$ (Fig. $4 \mathrm{~b}$ and Supplementary Fig. 3). Binding of one sodium ion repositions $\mathrm{N} 313$, which at the same time optimizes the geometry of the other sodium site, in line with the observed cooperativity in sodium binding.

In the repositioned NMDGT region of Glt $\mathrm{T}_{\mathrm{Tk}}^{\mathrm{sub}} \mathrm{G} 316$ and T317 are located closer to TMS8 than in Glt ${ }_{\mathrm{Tk}}^{\text {apo }}$. The position of G316 is stabilized by a hydrogen bond to the side chain carboxamide of 
a

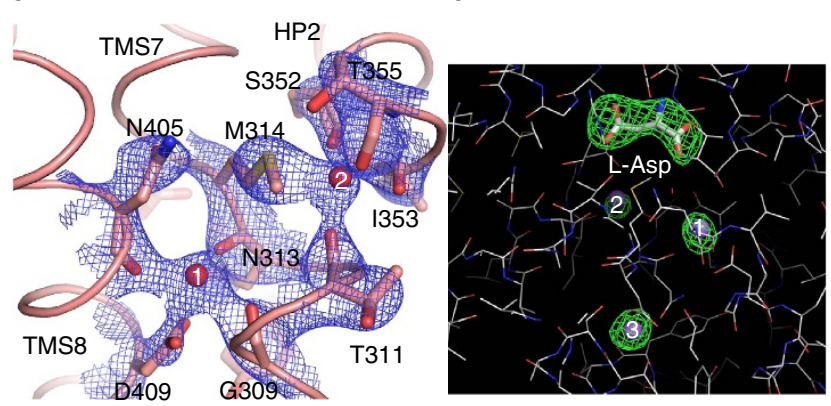

C

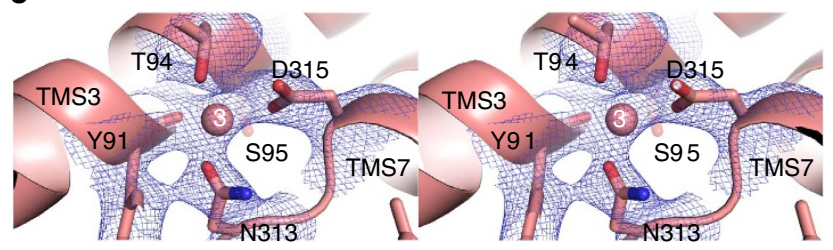

Figure 3 | Structure of Gltsk sodium binding sites. (a) Sodium binding sites $\mathrm{Na}^{+} 1$ and $\mathrm{Na}^{+}$2. The residues forming the sites are shown as sticks, sodium ions as spheres. The $2 \mathrm{fo}-\mathrm{fc}$ electron density is shown as blue mesh at $3 \sigma$. (b) Composite omit map (simulated annealing protocol) for the substrates L-Asp (in stick) and sodium ions (purple spheres), contoured at 3.5 $\sigma$. (c) Stereo-view of $\mathrm{Na}^{+} 3$ site, depicted as in a.
N405 in TMS 8, which in turn binds $\mathrm{Na}^{+} 1$ via its backbone carbonyl, again contributing to the strong coupling between the movement of the NMDGT region and sodium binding. The movement places T317 in an optimal position for aspartate binding, which provides a structural explanation for cooperativity between $\mathrm{Na}^{+}$and aspartate binding. In addition the movement of T317 forces the side chain of R401 to reorient, which creates space for aspartate binding, and places the guanidium group in an optimal orientation to interact with the $\beta$-carboxylate of aspartate. In this way sodium binding events in sites $\mathrm{Na}^{+} 1$ and $\mathrm{Na}^{+} 3$ not only interlink with each other, but also strongly affect the affinity for aspartate. This coupling is further reinforced because binding of sodium to the $\mathrm{Na}^{+} 3$ site leads to a movement of TMS3 (which contains three of the $\mathrm{Na}^{+} 3$ binding residues) towards TMS7, thereby pushing $\mathrm{T} 317$ towards the aspartate binding position (Fig. 4a).

After binding of $\mathrm{Na}^{+} 1, \mathrm{Na}^{+} 3$ and aspartate, $\mathrm{Na}^{+} 2$ is likely the last ion to bind ${ }^{9,13,25}$. The binding of $\mathrm{Na}^{+} 2$ is coupled to binding of the other two sodium ions and aspartate by the positioning of M314 at the center of the triangle formed by aspartate, $\mathrm{Na}^{+} 1$ and $\mathrm{Na}^{+} 2$ (Fig. $4 \mathrm{~b}$ ), and by the conformation of the tip of HP2, which interacts both with aspartate and with $\mathrm{Na}^{+} 2$. Only after binding of $\mathrm{Na}^{+} 2$ can the transport domain reorient to the inward-facing state, because $\mathrm{Na}^{+} 2$ binding closes the gate formed by the tip of HP2. MD simulations indeed show that the tip of HP2 is one of the most flexible regions of the protein, and that $\mathrm{Na}^{+} 2$ relatively easily leaves this site (Fig. 5 and Supplementary Note 1). a

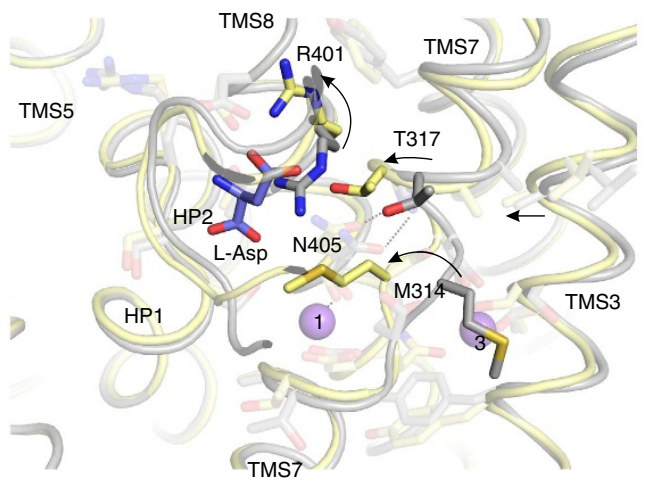

b

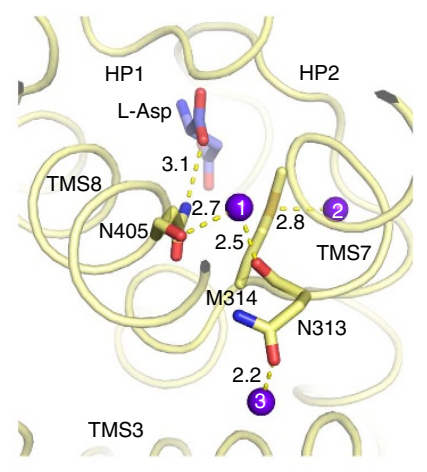

C

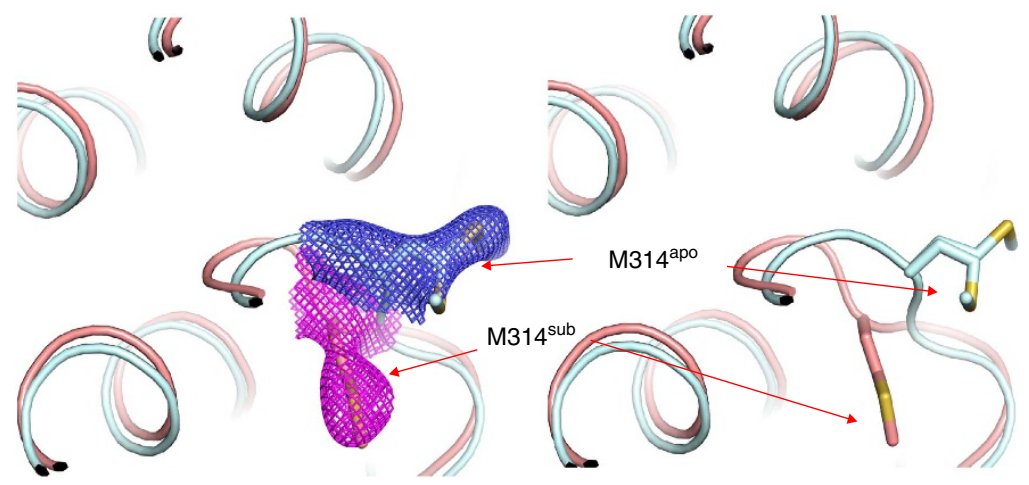

Figure 4 | Mechanism of sodium-aspartate coupling. (a) Superposition of Glt ${ }_{\mathrm{Tk}}^{\mathrm{sub}}$ (yellow) and Glt ${ }_{\mathrm{Tk}}^{\mathrm{apo}}$ (gray). Bound L-Asp shown as blue sticks and sodium ions as purple spheres. Arrows represent the movements during transition from apo to the substrate-bound state. Note that parts of the protein which are not directly involved in substrate-binding (for example, TMS2, TMS5) do not undergo any noticeable changes. (b) Interaction network between L-Asp (blue sticks) and sodium ions (purple spheres) mediated by N313, M314 and N405 (shown in sticks), distances are given in $\AA$ (also see Supplementary Fig. 3). (c) M314 in substrate-bound and substrate-free states with 2fo-fc electron density countered at $3 \sigma$ (left) and without density (right). In the apo state two alternative conformations of the side chain are resolved, consistent with the proposed inherent mobility of this residue. 


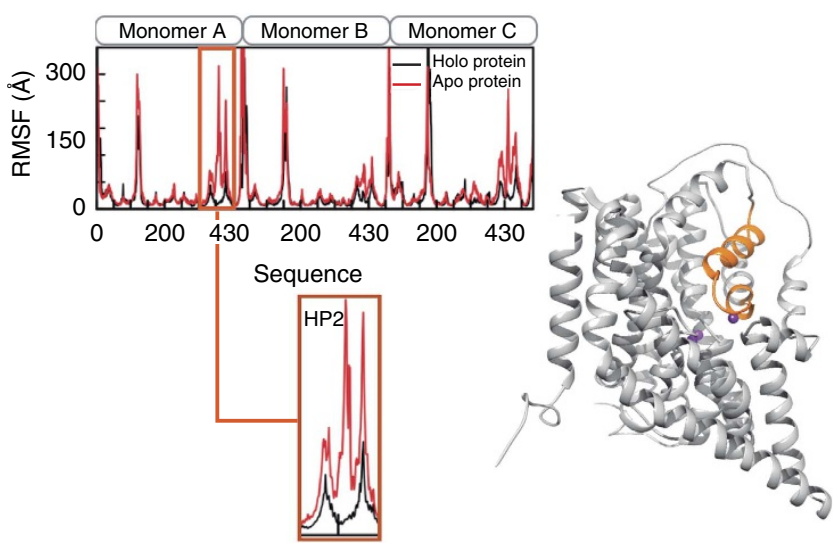

Figure 5 | HP2 displays enhanced local flexibility. Comparison of the protein atomic fluctuations averaged per residue in the presence/absence of the ligands in the binding site. Data was averaged from 100 ns MD simulations. The HP2 residues (in orange) involved in the gate opening showed enhanced local flexibility without the ligands (See also Supplementary Fig. 5 for RMSD values of the backbone).

\section{Discussion}

The crystal structures and MD simulations presented here provide insight in the coupled binding of three sodium ions and aspartate. The work is fully consistent with a model for transport in which the cooperative binding of two sodium ions $\left(\mathrm{Na}^{+} 1\right.$ and $\left.\mathrm{Na}^{+} 3\right)$ is followed by aspartate binding and finally binding of $\mathrm{Na}^{+} 2$. The order in which $\mathrm{Na}^{+} 1$ and $\mathrm{Na}^{+} 3$ bind may depend on an obligatory route of sodium ions from the $\mathrm{Na}^{+} 1$ site to the $\mathrm{Na}^{+} 3$ site $^{13}$. The postulation of an obligatory route was based on the structure of substrate-bound $\mathrm{Glt}_{\mathrm{Ph}}$, which showed that the $\mathrm{Na}^{+} 3$ site could be accessed only via the cavity of the $\mathrm{Na}^{+} 1$ site. Because the affinity of the $\mathrm{Na}^{+} 3$ site is higher than that of the $\mathrm{Na}^{+} 1$ site, the $\mathrm{Na}^{+} 3$ site would fill up before the $\mathrm{Na}^{+} 1$ site. Our data are consistent with this binding order, but comparison of the substrate-bound structure with that of the apo-protein (which was not available at the time of the previous simulations) suggests that substantial conformational changes in the NMDGT region must take place upon sodium binding. Neither the $\mathrm{Na}^{+} 1$ nor the $\mathrm{Na}^{+} 3$ site is fully formed in the apo state, and it is possible that an alternative access pathway may open upon repositioning of the NMDGT region and movements of the tip of HP2. In that case a random order of the binding of $\mathrm{Na}^{+} 1$ and $\mathrm{Na}^{+} 3$ may occur. Further simulations and experiments could clarify the issue. Finally, although the binding of three sodium ions to the sites identified here leads to a stable, crystallizable state, it is possible that also transient binding sites exist, which are briefly occupied while the $\mathrm{Na}^{+}$ions are en-route to their most stable positions ${ }^{14}$.

\section{Methods}

Protein expression and purification. E. coli MC1061 cells containing a pBAD24 derived plasmid for production of C-terminally $\mathrm{His}_{8}$-tagged $\mathrm{Glt}_{\mathrm{Tk}}$ were cultivated in $\mathrm{LB}$ medium $\left(37^{\circ} \mathrm{C}\right.$ and 200 r.p.m.), and expression was induced at an $\mathrm{OD}_{600}$ of 0.8 with $0.05 \% \mathrm{~L}$-arabinose for a period of $3 \mathrm{~h}$. Harvesting, membrane vesicle preparation, determination of total protein concentration and solubilisation in $\mathrm{n}$-Dodecyl- $\beta$-D-Maltopyranoside was done as described before ${ }^{12}$. To obtain apo-Glt $\mathrm{Tk}_{\mathrm{Tk}}$ we omitted $\mathrm{Na}^{+}$from all buffers. After solubilisation the solution centrifuged for $30^{\prime}$ at $4^{\circ} \mathrm{C}, 265,000 \mathrm{~g}$. The supernatant was incubated for $1 \mathrm{~h}$ on a rotating platform at $4{ }^{\circ} \mathrm{C}$ with $\mathrm{Ni}$-Sepharose (GE Healthcare, $0.5 \mathrm{ml}$ bed volume) preequilibrated with $50 \mathrm{mM}$ Tris $\mathrm{HCl} \mathrm{pH} 8,300 \mathrm{mM} \mathrm{KCl}, 0.15 \%$ n-Decyl- $\beta$-DMaltopyranoside (DM), $15 \mathrm{mM}$ imidazole $\mathrm{pH}$. The slurry was poured into a Poly-Prep chromatography column (BioRad), and washed with $10 \mathrm{ml}$ of $50 \mathrm{mM}$ Tris $\mathrm{HCl} \mathrm{pH} \mathrm{8,300} \mathrm{mM} \mathrm{KCl,} \mathrm{0.15 \%} \mathrm{DM,} 60 \mathrm{mM}$ Imidazole $\mathrm{pH} 8$. Glt $\mathrm{Tk}_{\mathrm{Tk}}$ was eluted with $50 \mathrm{mM}$ Tris $\mathrm{HCl} \mathrm{pH} \mathrm{8,} 300 \mathrm{mM} \mathrm{KCl}, 0.15 \% \mathrm{DM}, 500 \mathrm{mM}$ Imidazole $\mathrm{pH}$ 8, and further purified by size exclusion chromatography (SEC) on a Superdex 200 10/300 GL (GE Healthcare) column in equilibrated with buffer containing $10 \mathrm{mM}$ Hepes $\mathrm{KOH} \mathrm{pH} 8$, $100 \mathrm{mM} \mathrm{KCl}, 0.15 \%$ DM. After purification the protein concentration was determined
}

by UV absorption (NanoDrop), using the molecular weight and extinction coefficient calculated by the Expasy Protparam tool (web.expasy.org/protparam/). The protein was concentrated to $\sim 7 \mathrm{mg} \mathrm{ml}^{-1}$ using a spin concentrator (Vivaspin 2, 30,000 MWCO, PES membrane, Vivaproducts). For purification of the substrate-loaded protein solubilization and purification buffers contained $300 \mathrm{mM} \mathrm{Na}^{+}$instead of $\mathrm{K}^{+}$ and were supplemented with $10 \mu \mathrm{M}$ L-aspartate.

Isothermal titration calorimetry (ITC). ITC experiments were performed at a constant temperature of $25^{\circ} \mathrm{C}$ using an ITC200 calorimeter (MicroCal). $\mathrm{L}$-aspartate (dissolved in buffer containing $10 \mathrm{mM}$ Hepes $\mathrm{KOH} \mathrm{pH} 8,100 \mathrm{mM} \mathrm{KCl}$, $0.15 \% \mathrm{DM}$ and indicated sodium concentrations) was titrated into a thermally equilibrated ITC cell filled with $250 \mu \mathrm{l}$ of 3-20 $\mu \mathrm{M} \mathrm{Glt}_{\mathrm{Tk}}$ (concentration 13-15 $\mu \mathrm{M}$ ), dissolved the same buffer. Data were analysed using the ORIGIN-based software provided by MicroCal.

Crystallization, data collection and structure determination. Crystals of $\mathrm{Glt}_{\mathrm{Tk}}^{\mathrm{apo}}$ and $\mathrm{Glt}_{\mathrm{Tk}}^{\mathrm{sub}}$ were obtained with the vapour diffusion technique (hanging drop) with the following conditions: $25 \%$ glycerol/ PEG 4000, $100 \mathrm{mM}$ Tris/bicine, $\mathrm{pH} 8.5$, $60 \mathrm{mM} \mathrm{CaCl} 2 / \mathrm{MgCl}_{2}$ and $0.75-1 \%$ n-octyl- $\beta$-D-glucopyranoside (OG) and $20 \%$ PEG 400, 3\% Xylitol, $50 \mathrm{mM} \mathrm{MgCl}, 150 \mathrm{mM} \mathrm{NaCl}, 100 \mathrm{mM}$ Glycine pH 9 and $25 \%$ glycerol/ PEG 4000, $100 \mathrm{mM}$ Tris/bicine, $\mathrm{pH} 8.5,60 \mathrm{mM} \mathrm{CaCl} / \mathrm{MgCl}_{2}$, respectively. Crystals were flash-frozen in liquid nitrogen, cryo-protected with paraton- $\mathrm{N}$ and brought to the synchrotron for analysis. Data were collected at beam line X06SA (SLS, Villigen). In addition, we made many attempts to perform anomalous diffraction experiments via crystal soaking or co-crystallization with thallium salts but they did not yield any useful results.

Crystals of $\mathrm{Glt}_{\mathrm{Tk}}^{\mathrm{apo}}$ and Glt $\mathrm{Tk}_{\mathrm{Tk}}^{\mathrm{sub}}$ diffracted up to 2.5 and $2.6 \AA$ resolution; for Glt ${ }_{\mathrm{Tk}}^{\mathrm{apo}}$ it was not possible to collect fully complete dataset (overall completeness $79 \%$, see Table 1). Data were processed with XDS (ref. 27), and the structures were solved by Molecular Replacement with Phaser ${ }^{28}$ using previously published model of Glt $t_{\mathrm{Tk}}^{\mathrm{apo}}$ (PDB ID 4KY0). Manual rebuilding was done with COOT (ref. 29) and refinement with Phenix refine ${ }^{30}$. Refined models were deposited into PDB repository with the following IDs: 5DWY for $\mathrm{Glt}_{\mathrm{Tk}}^{\mathrm{apo}}$ and $5 \mathrm{E} 9 \mathrm{~S}$ for $\mathrm{Glt}_{\mathrm{Tk}}^{\mathrm{sub}}$ respectively. Images were prepared using Pymol (Schrödinger, LLC) and Ligplot + (ref. 31).

Molecular dynamics simulations. Two systems were simulated to assess the influence of the ligands in the dynamics of the $\mathrm{Glt}_{\mathrm{Tk}}$ : the holo system, which contained the three $\mathrm{Na}^{+}$ions and the aspartate in the binding site, and the same system, from which the ions and aspartate had been omitted. Atomistic MD simulations were carried out using the crystallographic model of $\mathrm{Glt}_{\mathrm{Tk}}$ (PDB code $4 \mathrm{KY} 0$ ) embedded in a lipid membrane with explicit solvent. To this purpose we used the CHARMM Membrane Builder ${ }^{32}$ and converted to Lipid14 PDB format ${ }^{33}$. Each of the original crystal structures was embedded in a lipid bilayer composed of 2501 palmitoyl-2-oleoylphosphatidylcholine (POPC) lipids, solvated in aqueous solution with TIP3P water molecules ${ }^{34}$ and $0.15 \mathrm{M} \mathrm{NaCl}$. The simulation systems contained a total of $\sim 170,000$ atoms. After the initial preparation, the systems were minimized and equilibrated as explained elsewhere ${ }^{33}$. After minimization, coordinates of the protein and ligands were fixed $\left(10 \mathrm{kcal} \mathrm{mol} \AA^{-2}\right)$ to allow for equilibration of the water and lipid densities, while increasing the temperature up to $303 \mathrm{~K}$. In a second equilibration phase, the systems were relaxed without restraints for $20 \mathrm{~ns}$. MD simulations were run with AMBER 14 (ref. 35, http://ambermd.org/) for another 80 ns using the NPT ensemble ( $1 \mathrm{~atm}, 303 \mathrm{~K})$ with periodic boundary conditions and the Langevin thermostat.

Free energy calculations. We calculated the relative binding free energy of $\mathrm{Na}^{+}$ ions at the $\mathrm{Na} 1, \mathrm{Na} 2$ and $\mathrm{Na} 3$ binding sites. In the case of $\mathrm{Na} 1$, we considered both the case where no other ions were bound, and the case with the Na3 site already filled. For Na2, assumed to be the last site occupied, all other ligands were included in their binding sites (Supplementary Table 2).

The standard binding free energy can be decomposed as a sum of the translocation and translational free energy contributions:

$$
\Delta G_{\mathrm{b}}=\Delta \Delta G_{\text {int }}+\Delta G_{\mathrm{tr}}
$$

where the first term represents the contribution due to the difference in interaction energy of the ligand upon translocation from the bulk to the binding site while the second term represents the change in the translational energy upon binding. The latter is associated to the local fluctuations of the unrestrained $\mathrm{Na}^{+}$bound in the binding site ${ }^{15,22,36}$, and was computed according to Heinzelmann et al. ${ }^{22}$.

The first term, the free energy of translocation, is calculated using thermodynamic integration (TI) by transforming the bound $\mathrm{Na}^{+}$into a water molecule while transforming another water molecule into a $\mathrm{Na}^{+}$ion in the bulk. The transformation is done in three steps: first a decharging step, then a change in van der Waals and bonded interactions, and finally a recharging step. For each step, we used 11 values of $\lambda$ to go from 0 to 1 by increments of 0.1 . Both a forward and a backward transformation were performed. At each $\lambda$ window, the system was first equilibrated for $0.5 \mathrm{~ns}$ and sampled for $1 \mathrm{~ns}$. In all cases, the last snapshot of the unrestrained MD equilibration process was used as initial coordinates for the TI calculations. Calculation of the free energy can run into convergence issues unless the ligand is restrained to the protein through anchoring atoms in the binding site. We therefore 
used harmonic distance restraints between the sodium ion and the surrounding protein residues in the binding site. We verified that the effect of these conformational restraints was negligible (in the case of the $\mathrm{Na} 1$ and $\mathrm{Na} 3$ binding sites around $0.003 \mathrm{kcal} \mathrm{mol}^{-1}$, and around $0.60 \mathrm{kcal} \mathrm{mol}^{-1}$ for the $\mathrm{Na} 2$ binding site).

The convergence of the calculations was assessed by monitoring the cumulative averages of the free energy differences (Supplementary Fig. 4). Given the smooth curves obtained, the free energy change associated to the transformation was calculated using the trapezoidal rule. Translocation free energies were calculated by averaging the values of the forward and backward transformations.

Data availability. All relevant data are available from the corresponding author upon reasonable request. The coordinates of the refined models and structure factors have been deposited into the PDB repository: 5DWY for $\mathrm{Glt}_{\mathrm{Tk}}^{\mathrm{apo}}$ and 5E9S for Glt $\mathrm{Tk}_{\mathrm{Tk}}^{\mathrm{sub}}$. The $\mathrm{Gl}_{\mathrm{TK}}^{\mathrm{apo}}$ structure (PDB ID 4KY0) was used as a molecular replacement model and for MD simulations.

\section{References}

1. Vandenberg, R. J. \& Ryan, R. M. Mechanisms of glutamate transport. Physiol. Rev. 93, 1621-1657 (2013).

2. Grewer, C., Gameiro, A. \& Rauen, T. SLC1 glutamate transporters. Pflugers Arch-Eur. J. Physiol. 466, 3-24 (2013).

3. Owe, S. G., Marcaggi, P. \& Attwell, D. The ionic stoichiometry of the GLAST glutamate transporter in salamander retinal glia. J. Physiol. 577, 591-599 (2006).

4. Zerangue, N. \& Kavanaugh, M. P. Flux coupling in a neuronal glutamate transporter. Nature 383, 634-637 (1996).

5. Levy, L. M., Warr, O. \& Attwell, D. Stoichiometry of the glial glutamate transporter GLT-1 expressed inducibly in a Chinese hamster ovary cell line selected for low endogenous $\mathrm{Na}^{+}$-dependent glutamate uptake. J. Neurosci. 18, 9620-9628 (1998).

6. Yernool, D., Boudker, O., Jin, Y. \& Gouaux, E. Structure of a glutamate transporter homologue from Pyrococcus horikoshii. Nature 431, 811-818 (2004).

7. Reyes, N., Ginter, C. \& Boudker, O. Transport mechanism of a bacterial homologue of glutamate transporters. Nature 462, 880-885 (2009).

8. Jensen, S., Guskov, A., Rempel, S., Hänelt, I. \& Slotboom, D. J. Crystal structure of a substrate-free aspartate transporter. Nat. Struct. Mol. Biol. 20, 1224-1226 (2013).

9. Boudker, O., Ryan, R. M., Yernool, D., Shimamoto, K. \& Gouaux, E. Coupling substrate and ion binding to extracellular gate of a sodium-dependent aspartate transporter. Nature 445, 387-393 (2007).

10. Verdon, G., Oh, S., Serio, R. N. \& Boudker, O. Coupled ion binding and structural transitions along the transport cycle of glutamate transporters. Elife 3, e02283 (2014).

11. Ryan, R. M., Compton, E. L. R. \& Mindell, J. A. Functional characterization of a $\mathrm{Na}^{+}$-dependent aspartate transporter from Pyrococcus horikoshii. J. Biol. Chem. 284, 17540-17548 (2009)

12. Groeneveld, M. \& Slotboom, D. J. Na ${ }^{(+)}$:aspartate coupling stoichiometry in the glutamate transporter homologue $\mathrm{Glt}(\mathrm{Ph})$. Biochemistry 49, 3511-3513 (2010).

13. Huang, Z. \& Tajkhorshid, E. Identification of the third $\mathrm{Na}^{+}$site and the sequence of extracellular binding events in the glutamate transporter. Biophysics 99, 1416-1425 (2010).

14. Larsson, H. P. et al. Evidence for a third sodium-binding site in glutamate transporters suggests an ion/substrate coupling model. Proc. Natl Acad. Sci. USA 107, 13912-13917 (2010).

15. Bastug, T. et al. Position of the third $\mathrm{Na}^{+}$site in the aspartate transporter $\mathrm{GltPh}$ and the human glutamate transporter, EAAT1. PLoS ONE 7, e33058-10 (2012).

16. Compton, E. L. R., Taylor, E. M. \& Mindell, J. A. The 3-4 loop of an archaeal glutamate transporter homolog experiences ligand-induced structural changes and is essential for transport. Proc. Natl Acad. Sci. USA 107, 12840-12845 (2010).

17. Mulligan, C. \& Mindell, J. A. Mechanism of transport modulation by an extracellular loop in an archaeal excitatory amino acid transporter (EAAT) homolog. J. Biol. Chem. 288, 35266-35276 (2013).

18. Tao, Z., Zhang, Z. \& Grewer, C. Neutralization of the aspartic acid residue Asp-367, but not Asp-454, inhibits binding of $\mathrm{Na}^{+}$to the glutamate-free form and cycling of the glutamate transporter EAAC1. J. Biol. Chem. 281, 10263-10272 (2006).

19. Tao, Z. et al. Mechanism of cation binding to the glutamate transporter EAAC1 probed with mutation of the conserved amino acid residue Thr101. J. Biol. Chem. 285, 17725-17733 (2010).

20. Zarbiv, R., Grunewald, M., Kavanaugh, M. P. \& Kanner, B. I. Cysteine scanning of the surroundings of an alkali-ion binding site of the glutamate transporter GLT-1 reveals a conformationally sensitive residue. J. Biol. Chem. 273, 14231-14237 (1998)

21. Silverstein, N., Crisman, T. J., Forrest, L. R. \& Kanner, B. I. Cysteine scanning mutagenesis of transmembrane helix 3 of a brain glutamate transporter reveals two conformationally sensitive positions. J. Biol. Chem. 288, 964-973 (2013).
22. Heinzelmann, G., Bastug, T. \& Kuyucak, S. Free energy simulations of ligand binding to the aspartate transporter $\mathrm{Glt}(\mathrm{Ph})$. Biophys. J. 101, 2380-2388 (2011).

23. Reyes, N., Oh, S. \& Boudker, O. Binding thermodynamics of a glutamate transporter homolog. Nat. Struct. Mol. Biol. 20, 634-640 (2013).

24. Hänelt, I., Jensen, S., Wunnicke, D. \& Slotboom, D. J. Low affinity and slow $\mathrm{Na}^{+}$binding precedes high affinity aspartate binding in the secondary-active transporter GltPh. J. Biol. Chem. 290, 15962-15972 (2015).

25. Venkatesan, S. et al. Refinement of the Central Steps of Substrate Transport by the Aspartate Transporter GltPh: Elucidating the Role of the Na2 Sodium Binding Site. PLoS Comput. Biol. 11, e1004551 (2015).

26. Lolkema, J. S. \& Slotboom, D. J. The Hill analysis and co-ion-driven transporter kinetics. J. Gen. Physiol. 145, 565-574 (2015).

27. Kabsch, W. Integration, scaling, space-group assignment and post-refinement. Acta Crystallogr. D Biol. Crystallogr 66, 133-144 (2010).

28. McCoy, A. J. et al. Phaser crystallographic software. J. Appl. Crystallogr. 40, 658-674 (2007).

29. Emsley, P., Lohkamp, B., Scott, W. G. \& Cowtan, K. Features and development of Coot. Acta Crystallogr. D Biol. Crystallogr 66, 486-501 (2010).

30. Afonine, P. V. et al. Towards automated crystallographic structure refinement with phenix.refine. Acta Crystallogr. D Biol. Crystallogr 68, 352-367 (2012).

31. Laskowski, R. A. \& Swindells, M. B. LigPlot ${ }^{+}$: multiple ligand-protein interaction diagrams for drug discovery. J. Chem. Inf. Model. 51, 2778-2786 (2011).

32. Jo, S., Lim, J. B., Klauda, J. B. \& Im, W. CHARMM-GUI Membrane Builder for mixed bilayers and its application to yeast membranes. Biophys. J. 97, 50-58 (2009).

33. Dickson, C. J. et al. Lipid14: the amber lipid force field. J. Chem. Theory Comput 10, 865-879 (2014).

34. Jorgensen, W. L., Chandrasekhar, J., Madura, J. D., Impey, R. W. \& Klein, M. L. Comparison of simple potential functions for simulating liquid water. J. Chem. Phys. 79, 926-935 (1983).

35. Case, D. A. et al. Amber 14, University of California, San Francisco (2014).

36. Karplus, M. \& Kushick, J. N. Method for estimating the configurational entropy of macromolecules. Macromolecules 14, 325-332 (1981).

\section{Acknowledgements}

We thank A.J.M. Driessen for use of his ITC machine. The research leading to these results received funding from the European Community's Seventh Framework Programme (FP7/2007-2013) under BioStruct-X (to D.J.S. and A.G., grant agreement 283570). This work was funded by the Netherlands Organisation for Scientific Research (NWO) (NWO ECHO grant 711.011.001 and NWO Vici grant 865.11.001 to D.J.S.) and the European Research Council (ERC) (ERC Starting Grant 282083 to D.J.S.). The European Synchrotron Radiation Facility (ESRF) and the Swiss Light Source (SLS) are acknowledged for beamline facilities.

\section{Author contributions}

All authors designed experiments and simulations, analysed data and wrote the manuscript, A.G. and S.J. performed experiments, I.F. performed simulations.

\section{Additional information}

Supplementary Information accompanies this paper at http://www.nature.com/ naturecommunications

Competing financial interests: The authors declare no competing financial interests.

Reprints and permission information is available online at http://npg.nature.com/ reprintsandpermissions/

How to cite this article: Guskov, A. et al. Coupled binding mechanism of three sodium ions and aspartate in the glutamate transporter homologue Glt $_{\mathrm{Tk}}$. Nat. Commun. 7, 13420 doi: 10.1038/ncomms13420 (2016).

Publisher's note: Springer Nature remains neutral with regard to jurisdictional claims in published maps and institutional affiliations.

This work is licensed under a Creative Commons Attribution 4.0 International License. The images or other third party material in this article are included in the article's Creative Commons license, unless indicated otherwise in the credit line; if the material is not included under the Creative Commons license, users will need to obtain permission from the license holder to reproduce the material. To view a copy of this license, visit http://creativecommons.org/licenses/by/4.0/

(C) The Author(s) 2016 